\title{
Reduction in choroidal thickness of macular area in polypoidal choroidal vasculopathy patients after intravitreal ranibizumab therapy
}

\author{
Tadayuki Nishide • Natsuki Hayakawa $\cdot$ Misako Nakanishi • \\ Mai Ishii • Shinya Okazaki • Ikuko Kimura • \\ Etsuko Shibuya • Nobuhisa Mizuki
}

Received: 22 January 2013 / Revised: 13 June 2013 / Accepted: 27 June 2013 /Published online: 18 July 2013

(C) The Author(s) 2013. This article is published with open access at Springerlink.com

\begin{abstract}
Background To evaluate changes in retinal and choroidal thickness changes after three intravitreal ranibizumab (IVR) injections for polypoidal choroidal vasculopathy (PCV) using enhanced depth-imaging-optical coherence tomography (EDI-OCT).

Methods In this retrospective, observational case series, EDI-OCT was used to measure changes in choroidal thickness at nine points in a lattice shape in the macula before and after introductory-stage IVR.

Results Choroidal thickness was decreased at all nine points in the lattice shape, but was significantly decreased only at the fovea.

Conclusion The subfoveal choroidal thickness may be reduced by introductory-stage IVR in patients with PCV. In particular, choroidal thickness at the fovea was reduced during the early stage of treatment.
\end{abstract}

Keywords Choroidal thickness $\cdot$ Polypoidal choroidal vasculopathy · Enhanced depth imaging · Optical coherence tomography $\cdot$ Ranibizumab

\section{Introduction}

Exudative age-related macular degeneration (AMD) is a disease in which neovessels and vascular anomalies form in the macula. Polypoidal choroidal vasculopathy (PCV) of

T. Nishide $(\bowtie) \cdot$ N. Hayakawa $\cdot$ M. Nakanishi $\cdot$ M. Ishii $\cdot$

S. Okazaki $\cdot$ I. Kimura $\cdot$ E. Shibuya $\cdot$ N. Mizuki

Department of Ophthalmology and Visual Science, Yokohama City

University Graduate School of Medicine, 3-9, Fukuura,

Kanazawa-ku, Yokohama-city, Kanagawa, Japan 236-0004

e-mail: nishide@abox3.so-net.ne.jp subtype of AMD has neovessels and vascular anomalies in not only the fovea but also the macula. Optical coherence tomography (OCT) of the fovea alone cannot identify changes at lesion sites. Among the many OCT methods of assessing AMD are enhanced depth-imaging-OCT (EDI-OCT) [1-5]. Although several studies have evaluated subfoveal choroidal thickness in patients with AMD, subfoveal assessment alone is insufficient in patients with PCV, a disease that originates in both the subfoveal and extrafoveal choroid and later spreads throughout the choroid. Since PCV involves the formation of network vessels and accompanying polypoidal vessels, analysis of both subfoveal and extrafoveal choroidal thickness is necessary, especially in assessing treatment outcomes.

In contrast to standard OCT imaging, EDI-OCT uses long wavelengths to achieve high penetration [6]. With EDI-OCT, the retinal pigment epithelium is highly permeable, and high resolution is achieved at close to optimal distance from the light source. The Heidelberg Engineering Spectralis OCT utilizes these characteristics to allow OCT to be applied at even closer distances. This puts the choroid at the optimal distance from the light source, produces an upside-down image, and projects the choroid clearly. Thus, in a normal eye, the choroid will be the thickest at the fovea, thicker temporal to the fovea than nasal, and thicker superior to the fovea than inferior. Choroidal thickness has been found to decrease with age and to be inversely related to the axial length of the eye. Moreover, a thinner choroid has been associated with refractive error tending more towards myopia [7-12].

Ranibizumab (Lucentis ${ }^{\circledR}$, Novartis) is a humanized monoclonal antibody fragment that binds to vascular endothelial growth factor (VEGF) isoforms and has been approved for the treatment of patients with wet AMD [13, 14]. We retrospectively analyzed choroidal thickness at nine sites in the macula using EDI-OCT before and after the introductory stage of intravitreal ranibizumab $(0.5 \mathrm{mg})$ therapy (IVR), performed 


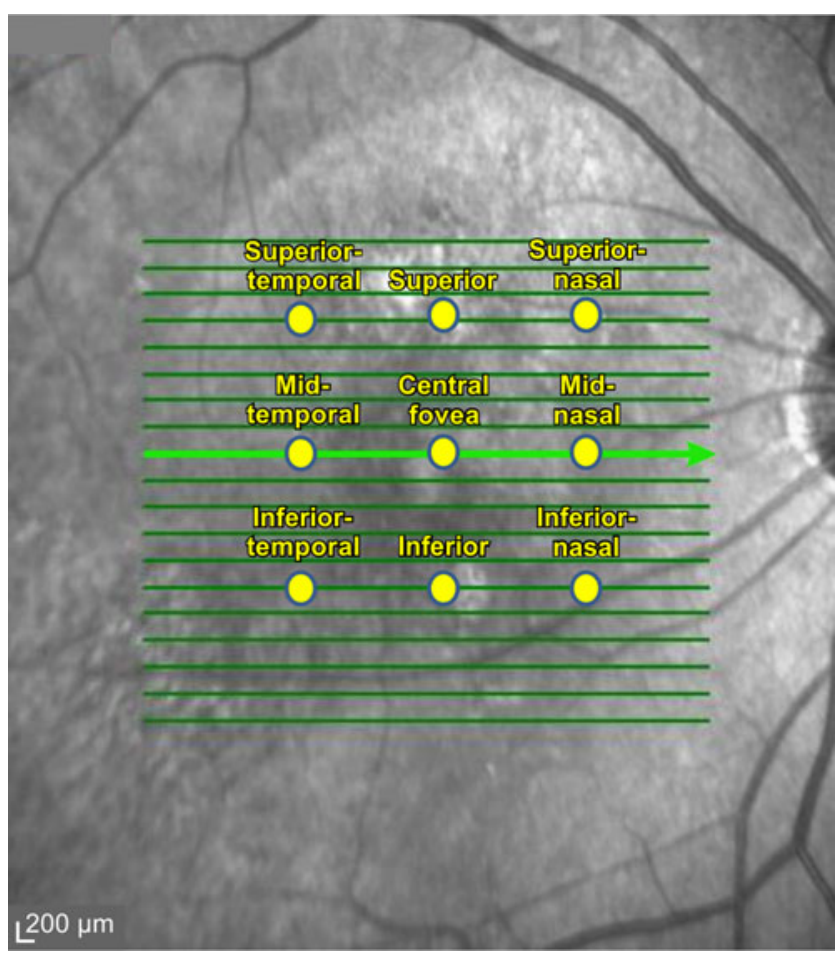

Fig. 1 The nine measurement sites of choroidal thickness using crosssectional images of enhanced depth-imaging-optical coherence tomography. This photograph is obtained in volume mode of Spectralis OCT

once per month for three consecutive months, in patients with PCV [14].

We investigated in which part the biggest change of choroidal thickness of macula area after ranibizumab therapy would appear in PCV.

\section{Materials and methods}

This study is a retrospective and observational case series. This study adhered to the tenets of the Declaration of Helsinki and was given exempt status by the Yokohama City
University institutional review board. Here, we examine medical data that was previously collected. Because this study was retrospective and all data were de-identified, there was no risk incurred by subjects who included their private health information in analyses.

All subjects were examined in an outpatient setting at the Yokohama City University Eye Clinic. Choroidal thickness

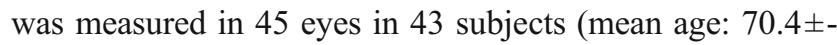
8.0 years), including 33 eyes in 31 males and 12 eyes in 12 females, who had been diagnosed with PCV and who had not undergone IVR treatment for at least 1 year. There was no patient who underwent treatment for PCV other than IVR. Patients with diseases that affect choroidal thickness, including high myopia and uveitis, were excluded. EDI-OCT was performed using a Spectralis (Heidelberg Engineering) OCT before and after the introductory stage of IVR, consisting of IVRs performed once monthly for 3 months.

Choroidal thickness, defined as the distance from the inferior margin of the retinal pigment epithelium to the choroid/sclera border, was measured at nine sites in the macula. These nine sites formed a lattice shape with the fovea at the center, with pairs of sites 1,200 $\mu \mathrm{m}$ apart (Fig. 1). These nine sites included points superior-nasal, superior, superior-temporal, mid-nasal, central, mid-temporal, inferior-nasal, inferior, and inferiortemporal to the fovea. Changes in choroidal thickness were calculated from before to after IVR. One unblinded investigator and two blinded investigators to treatment status determined the choroidal thickness. The average outcome of three measurements of the choroidal thickness was used for calculation. None of the patients experienced any complications, such as vitreous hemorrhage and endophthalmitis.

\section{Results}

No patient dropped out during the introductory stage of IVR. Pre- and post-IVR thicknesses and changes in thickness are shown in the Table 1. Choroidal thickness was decreased at
Table 1 Mean \pm SD foveal thickness at nine macular sites before and after introductorystage IVR and mean change in thickness in 45 eyes of 43 patients with PCV

\begin{tabular}{llll}
\hline Macular site & Pre-IVR thickness $(\mu \mathrm{m})$ & Post-IVR thickness $(\mu \mathrm{m})$ & $\begin{array}{l}\text { Change in thickness } \\
(\mu \mathrm{m} / \mathrm{month})\end{array}$ \\
\hline Superior-nasal & $223.6 \pm 64.5$ & $210.8 \pm 70.6$ & 0.96 \\
Superior & $240.7 \pm 67.3$ & $231.9 \pm 84.5$ & 0.97 \\
Superior-temporal & $225.2 \pm 60.2$ & $207.5 \pm 67.7$ & 0.94 \\
Mid-nasal & $218.0 \pm 57.8$ & $190.9 \pm 64.8$ & 0.90 \\
Central fovea & $243.2 \pm 65.6$ & $213.2 \pm 74.5$ & $0.89(p=0.03)$ \\
Mid-temporal & $226.9 \pm 57.1$ & $209.1 \pm 72.9$ & 0.93 \\
Inferior nasal & $216.0 \pm 61.9$ & $202.3 \pm 69.9$ & 0.94 \\
Inferior & $233.4 \pm 76.4$ & $221.1 \pm 90.9$ & 0.94 \\
Inferior temporal & $218.1 \pm 69.4$ & $201.4 \pm 73.9$ & 0.92 \\
\hline
\end{tabular}



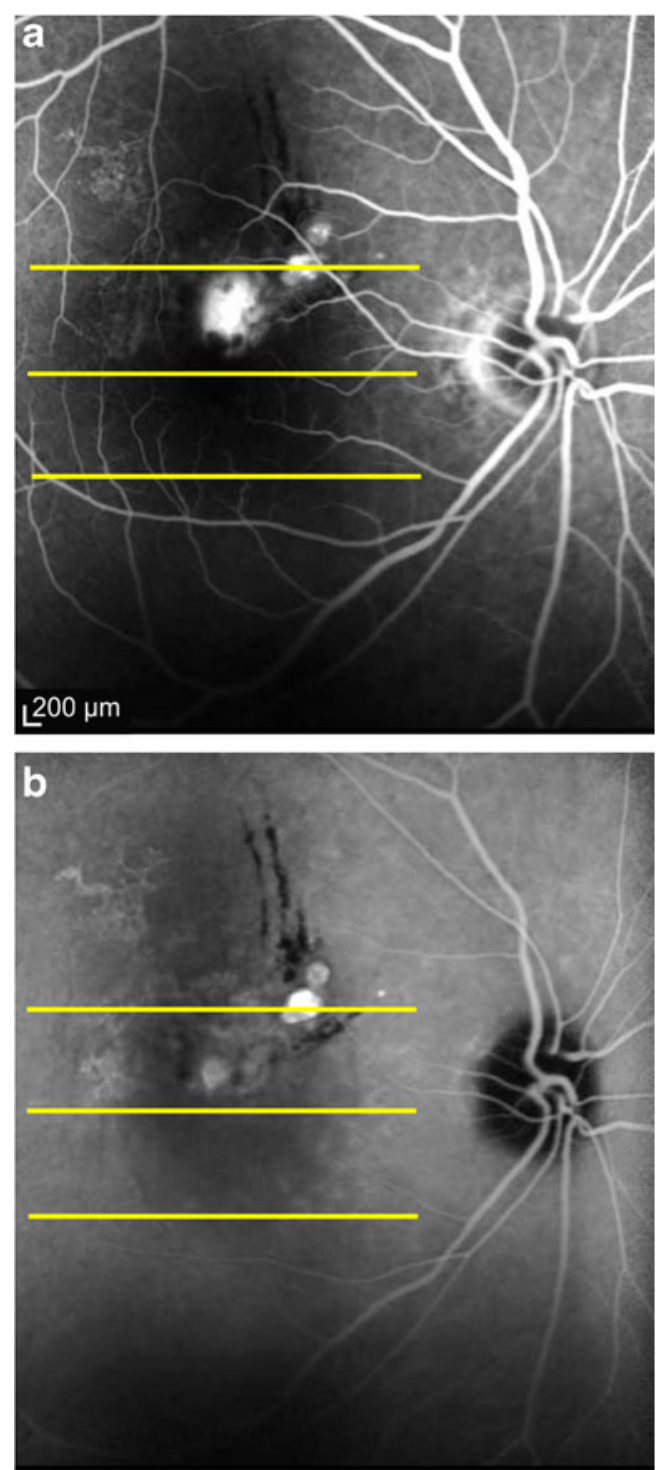

Fig. 2 a Angiographic findings using fluorescein before intravitreal ranibizumab therapy in Case b Angiographic findings using indocyanine green before intravitreal ranibizumab therapy in Case. Yellow lines indicate where choroidal thickness was measured using enhanced depth-imaging-optical coherence tomography

all sites, with significant decreases in the central fovea ( $p=0.03$; Table 1). The mean rate of change at the nine sites was $-0.93 \pm 0.03 \mu \mathrm{m} / \mathrm{month}$. In addition, the pre-to-post IVR introduction stage rate of change at the central fovea was found to be statistically significant on the Mann-Whitney $U$ test analysis. In the IVR treatment for PCV, post-treatment choroidal thickness was decreased at all nine measurement sites compared to pre-treatment choroidal thickness. In addition, a statistically significant decrease was observed in subfoveal choroidal thickness. These results suggest the possibility that choroidal thickness throughout the macula is reduced from pre- to post-IVR introduction-stage therapy in PCV. a
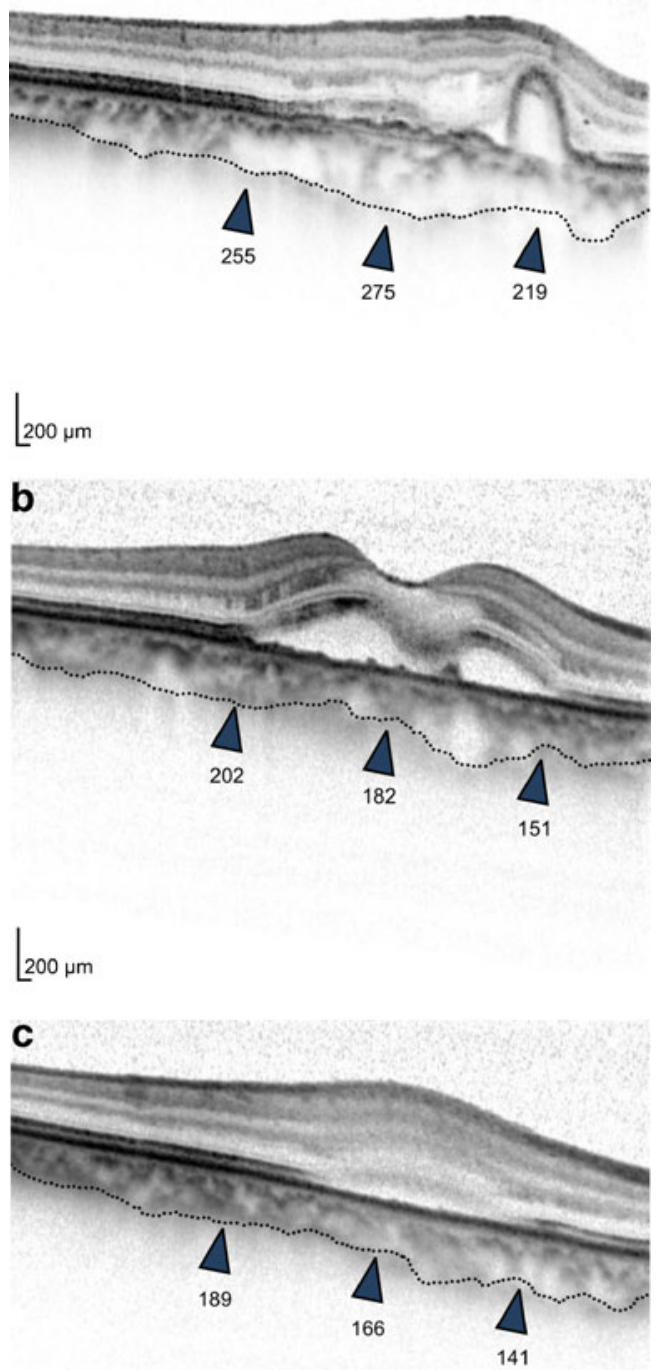

$200 \mu \mathrm{m}$

Fig. 3 a, b, c Choroidal thickness measurements on enhanced depthimaging-optical coherence tomography images before intravitreal ranibizumab therapy in Case. Labels represent the choroidal thickness (in $\mu \mathrm{m}$ ) at the location indicated by the arrowheads. The superior measurement line (Fig. 3a), central measurement line (Fig. 3b), and inferior measurement lines (Fig. 3c) are shown. The broken line shows the posterior border of the choroid

We showed the representative case of PCV whose choroidal thickness at the fovea was reduced during the early stage of IVR treatment.

A 68-year-old female became aware of distorted vision in her right eye; a fundoscopic examination revealed an orangered protrusion. The patient had polypoidal vessels in the vicinity of the site superior to the fovea, and PED was observed using fluorescein (Fig. 2a) and using indocyanine green (Fig. 2b). She was diagnosed with PCV based on a contrast study and was treated with three consecutive 

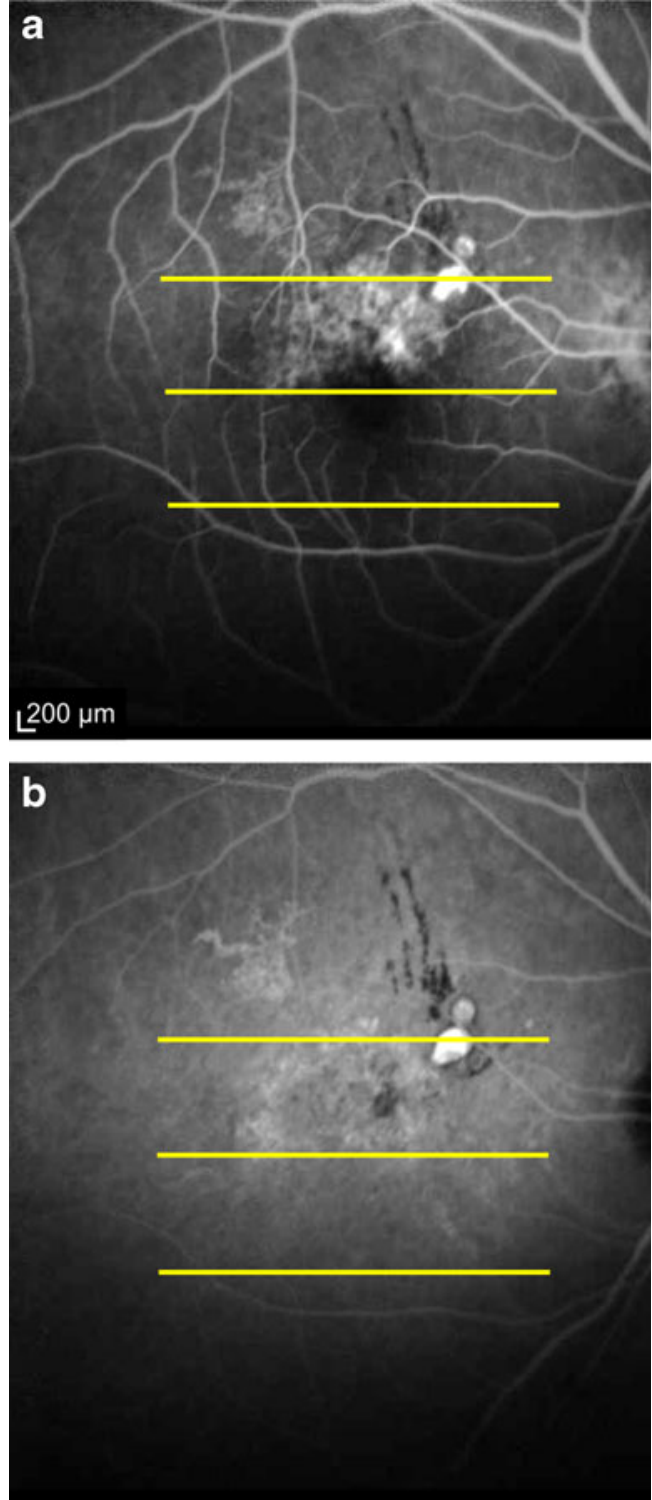

Fig. 4 a Angiographic findings using fluorescein after intravitreal ranibizumab therapy in Case $\mathbf{b}$ Angiographic findings using indocyanine green after intravitreal ranibizumab therapy in Case. Yellow lines indicate where choroidal thickness was measured using enhanced depth-imaging-optical coherence tomography

monthly IVRs. Prior to treatment, the choroidal thicknesses at the superior-nasal, superior, and superior-temporal sites were $219 \mu \mathrm{m}, 275 \mu \mathrm{m}$, and $255 \mu \mathrm{m}$ (Fig. 3a); mid-nasal, central fovea, and mid-temporal sites were $151 \mu \mathrm{m}, 182 \mu \mathrm{m}$, and $202 \mu \mathrm{m}$ (Fig. 3b); inferior-nasal, inferior, and inferiortemporal sites were $141 \mu \mathrm{m}, 166 \mu \mathrm{m}$, and $189 \mu \mathrm{m}$ (Fig. 3c). A contrast study following the three IVRs found reduced activity at the lesion site observed using fluorescein (Fig. 4a) and using indocyanine green (Fig. 4b). The choroidal thicknesses at the superior-nasal, superior, and superior-temporal sites were $206 \mu \mathrm{m}, 265 \mu \mathrm{m}$, and $237 \mu \mathrm{m}$ (Fig. 5a); mid-nasal, central fovea, and mid-temporal sites were $146 \mu \mathrm{m}, 169 \mu \mathrm{m}$, and $196 \mu \mathrm{m}$ (Fig. 5b); inferior-nasal, inferior, and inferior-
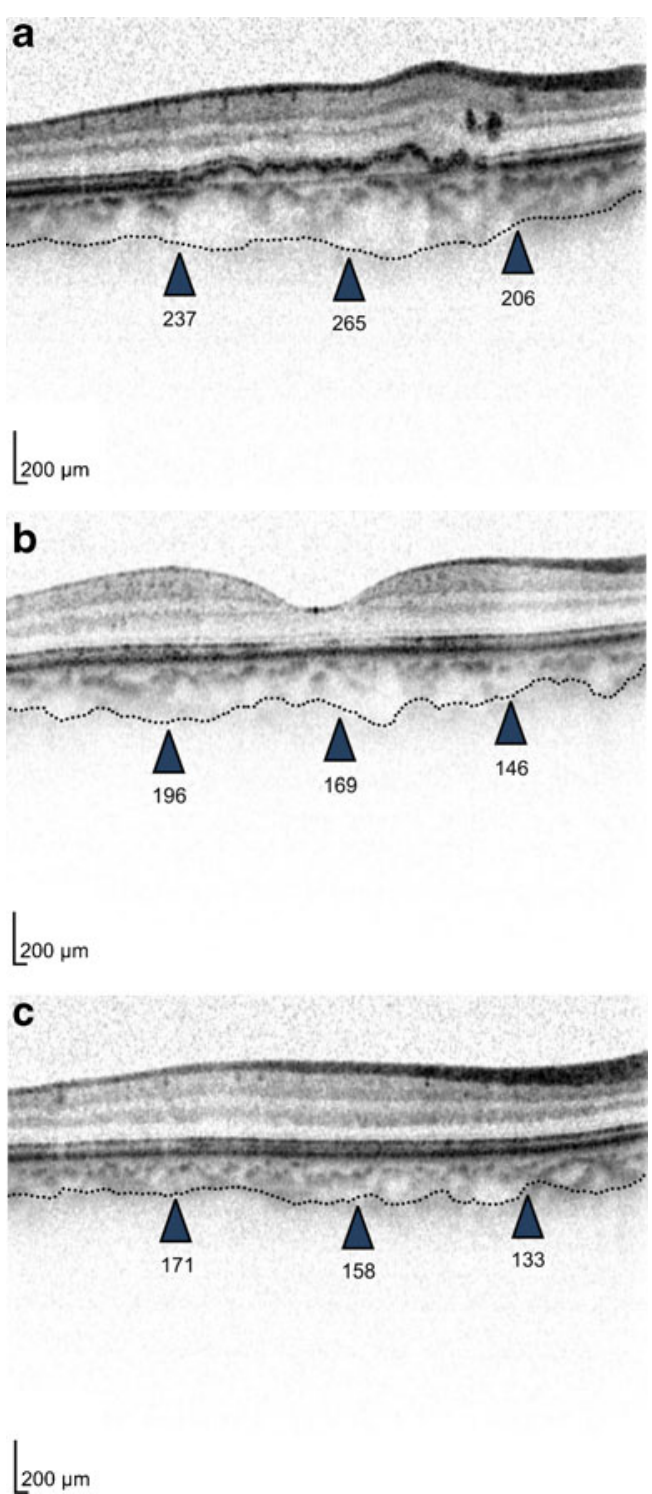

Fig. 5 a, b, c Enhanced depth-imaging-optical coherence tomography images after intravitreal ranibizumab therapy in Case. Labels represent the choroidal thickness (in $\mu \mathrm{m}$ ) at the location indicated by the arrowheads. The superior measurement line (Fig. 5a), central measurement line (Fig. 5b), and inferior measurement lines (Fig. 5c) are shown. The broken line shows the posterior border of the choroid

temporal sites were $133 \mu \mathrm{m}, 158 \mu \mathrm{m}$, and $171 \mu \mathrm{m}$ (Fig. 5c). The pre-treatment visual acuity in her right eye was $20 / 50$, while her post-treatment visual acuity improved to 20/25.

\section{Discussion}

We have studied changes in choroidal thickness occurring after introductory IVR in patients with PCV using EDI-OCT. The average choroid thickness decreased at all nine sites, with the rate of change of choroidal thickness ranging from -0.89 to $-0.97 \mu \mathrm{m} / \mathrm{month}$. The highest rate of change was at the 
central fovea, with this change being statistically significant. These findings indicate that introductory IVR treatment contributed to decreased subfoveal choroidal thickness.

Three subtypes of AMD have been described, typical AMD, retinal angiomatous proliferation (RAP), and PCV. PCV spreads throughout the choroid, with network and polypoidal vessels present in the choroid. Reduced dilation of medium and large choroidal vessels, as well as reduced enlargement of the choroidal stroma, may have contributed to the change in choroidal thickness we observed.

We analyzed the change in the choroidal thickness that was found after introductory IVR treatment. Patients require different numbers of IVR administrations to stabilize PCV; therefore, comparisons at PCV stabilization would have involved large differences in ranibizumab doses among patients. Rather than comparing choroids that had stabilized after the end of the treatment, we assessed choroids after 3 IVRs, when doses and administration periods were the same for all patients.

The choroid supplies oxygen and energy to the outer layers of the retina and dissipates any heat generated by retinal activity. Choroid activity is thought to be higher in the macula, particularly in and near the fovea, than elsewhere in the retina. PCV results in edema throughout the macular choroid. IVR is thought to promote partial recovery of choroidal function, dilate choroidal vessels, and improve stromal edema. In particular, introductory IVR has been found to strongly improve stromal edema in and near the fovea, where physiological activity is high. We could not determine whether the decrease in choroidal thickness we observed improved visual function [15]. However, the reduction of choroidal enlargement indicates an indirect suppression of disease activity; therefore, the recovery of normal choroidal thickness would likely have positive effects on visual function. Furthermore, introductory IVR has been shown to suppress the release of cytokines at lesion sites in the choroid, thereby reducing choroidal thickness regardless of whether abnormal vascular networks or polypoidal vessels were present.

Although we evaluated the effects of introductory IVR on choroidal thickness, many of the patients we assessed went on to receive chronic-stage IVR treatment. It is therefore necessary to conduct long-term studies to confirm whether the improvements in choroidal thickness in and near the fovea were maintained. Moreover, future studies should assess the effects of introductory-stage IVR on choroidal thickness in patients with AMD and RAP and to investigate whether changes in choroidal thickness are observed regardless of lesion location.

We detected the biggest change of choroidal thickness after ranibizumab therapy was appeared in fovea of macula area.

Acknowledgments I would like to express my very great appreciation to Professor Nobuhisa Mizuki for his valuable and constructive suggestions during the planning and development of this research work.
Funding/Support All authors have completed and submitted the ICMJE Form for Disclosure of Potential Conflicts of Interest.

Author contributions Design of study (T.N., N.H., M.N.); conduct of study (T.N., N.H., M.N., M.I., S.O., I.K., E.S., N.M.); analysis of data (T.N., N.H., M.N., M.I., S.O., E.S.); and manuscript preparation (T.N., N.H., M.N., N.M.).

Financial disclosures The authors report no financial disclosures.

Author statement The authors have full control of all primary data and they agree to allow Graefe's Archive for Clinical and Experimental Ophthalmology to review their data if requested.

Open Access This article is distributed under the terms of the Creative Commons Attribution License which permits any use, distribution, and reproduction in any medium, provided the original author(s) and the source are credited.

\section{References}

1. Spaide RF (2009) Enhanced depth imaging optical coherence tomography of retinal pigment epithelial detachment in age-related macular degeneration. Am J Ophthalmol 147(4):644-652

2. Gorczynska I, Srinivasan VJ, Vuong LN, Chen RW, Liu JJ, Reichel E, Wojtkowski M, Schuman JS, Duker JS, Fujimoto JG (2009) Projection OCT fundus imaging for visualising outer retinal pathology in non-exudative age-related macular degeneration. $\mathrm{Br} \mathrm{J}$ Ophthalmol 93(5):603-609

3. Querques G, Querques L, Forte R, Massamba N, Coscas F, Souied EH (2012) Choroidal changes associated with reticular pseudodrusen. Investig Ophthalmol Vis Sci 53(3):1258-1263

4. Rahman W, Chen FK, Yeoh J, Patel P, Tufail A, Da Cruz L (2011) Repeatability of manual subfoveal choroidal thickness measurements in healthy subjects using the technique of enhanced depth imaging optical coherence tomography. Investig Ophthalmol Vis Sci 52(5):2267-2271

5. Yeoh J, Rahman W, Chen F, Hooper C, Patel P, Tufail A, Webster AR, Moore AT, Dacruz L (2010) Choroidal imaging in inherited retinal disease using the technique of enhanced depth imaging optical coherence tomography. Graefes Arch Clin Exp Ophthalmol 248(12):1719 1728

6. Kodach VM, Kalkman J, Faber DJ, van Leeuwen TG (2010) Quantitative comparison of the OCT imaging depth at $1300 \mathrm{~nm}$ and $1600 \mathrm{~nm}$. Biomed Opt Express 1(1):176-185

7. Ikuno Y, Kawaguchi K, Nouchi T, Yasuno Y (2010) Choroidal thickness in healthy Japanese subjects. Investig Ophthalmol Vis Sci 51(4):2173-2176

8. Margolis R, Spaide RF (2009) A pilot study of enhanced depth imaging optical coherence tomography of the choroid in normal eyes. Am J Ophthalmol 147(5):811-815

9. Koizumi H, Yamagishi T, Yamazaki T, Kawasaki R, Kinoshita S (2011) Subfoveal choroidal thickness in typical age-related macular degeneration and polypoidal choroidal vasculopathy. Graefes Arch Clin Exp Ophthalmol 249(8):1123-1128

10. Fujiwara T, Imamura Y, Margolis R, Slakter JS, Spaide RF (2009) Enhanced depth imaging optical coherence tomography of the choroid in highly myopic eyes. Am J Ophthalmol 148(3):445-450

11. Imamura Y, Iida T, Maruko I, Zweifel SA, Spaide RF (2011) Enhanced depth imaging optical coherence tomography of the sclera in dome-shaped macula. Am J Ophthalmol 151(2):297-302

12. Ikuno Y, Maruko I, Yasuno Y, Miura M, Sekiryu T, Nishida K, Iida T (2011) Reproducibility of retinal and choroidal thickness 
measurements in enhanced depth imaging and high-penetration optical coherence tomography. Investig Ophthalmol Vis Sci 52(8):5536-5540

13. Kokame GT, Yeung L, Lai JC (2010) Continuous anti-VEGF treatment with ranibizumab for polypoidal choroidal vasculopathy: 6-month results. Br J Ophthalmol 94(3):297-301

14. Hikichi T, Ohtsuka H, Higuchi M, Matsushita T, Ariga H, Kosaka S, Matsushita R, Takami K (2010) Improvement of angiographic findings of polypoidal choroidal vasculopathy after intravitreal injection of ranibizumab monthly for 3 months. Am J Ophthalmol 150(5):674-682

15. Sogawa K, Nagaoka T, Takahashi A, Tanano I, Tani T, Ishibazawa A, Yoshida A (2012) Relationship between choroidal thickness and choroidal circulation in healthy young subjects. Am J Ophthalmol 153(6):1129-1132 